\title{
Perspectives de recherche en hydrologie urbaine
}

\section{DESBORDES}

Institut des sciences de l'ingénieur

de Montpellier

UMR Hydrosciences

Monpellier

Université Montpellier 2 34095 Montpellier Cedex 5 desbordes@isim.univmontp2.fr
L'hydrologie urbaine vise à l'étude des relations entre le cycle de l'eau et l'urbanisation. Née de la demande sociale en matière d'assainissement des agglomérations, elle s'est structurée scientifiquement durant les trente dernières années. Initialement consacrée à l'étude des flux d'eau résultant des précipitations, elle s'est par la suite intéressée aux flux de polluants associés à ces flux d'eau... Aujourd'hui, elle s'ouvre également aux sciences sociales. Elle offre ainsi de multiples possibilités de recherches dans des domaines variés parmi lesquels : la modélisation des écoulements de crue en milieu urbain, la gestion intégrée des systèmes d'assainissement (comprenant la gestion en temps réel de ces systèmes) dans un concept de développement durable, la conception d'ouvrages innovants s'inscrivant dans ce concept et prenant en compte les dimensions socioéconomiques et socioculturelles de Thydrologie urbaine.

Mots-clés ; hydrologie urbaine, assainissement, recherche.

\section{Potential researches in urban hydrology}

Urban hydrology is related to the water cycle in urban space. Suggested by the social demand regarding urban sewerage, its scientific aspects have been developed during the past thirty years. Dealing first with the study of urban rainfall-runoff process, it has been progressively related to the study of pollutions included in that process. Today, urban hydrology is also dealing with social sciences. Many research topics can be explored, among which : urban flooding modeling, urban rainfall measurement and modeling, integrated management of urban drainage systems (including real time control) and sustainable development, design of innovative facilities dealing with these concepts and taking into account socioeconomical and sociocultural aspects of urban hydrology.

Key words : urban hydrology, sewerage, research. 


\section{Introduction}

Depuis une trentaine d'années, l'hydrologie urbaine, discipline scientifique et technique traitant du cycle de l'eau en milieu urbain, a fait l'objet de nombreuses recherches à caractère fondamental ou finalisé. Initialement dédiée aux aspects physiques du cycle, elle s'est progressivement élargie vers des aspects variés faisant appel à de multiples disciplines scientifiques. Cette ouverture permet aujourd'hui d'énoncer sommairement les principaux secteurs susceptibles de connaître des développements dans les prochaines années.

\section{2}

\section{Objectifs et statut de l'hydrologie urbaine}

L'hydrologie urbaine est une discipline scientifique et technique dont la structuration scientifique s'est déroulée au cours des trente dernières années. Elle a pour objet l'étude des relations entre l'urbanisation et le cycle de l'eau. Elle donne ainsi lieu à des recherches à caractère fondamental, par exemple sur le rôle des (plaques urbaines » dans les échanges terre-atmosphère ou la génération de microclimats urbains. Elle donne également lieu à des recherches à caractère finalisé, visant à définir des méthodes et des techniques d'aménagement de l'espace destinées, simultanément, à protéger les agglomérations et leurs habitants contre les nuisances du cycle de l'eau (maladies, inondations) et à protéger les milieux hydriques récepteurs contre les nuisances engendrées par les agglomérations (pollutions, impacts hydrauliques...).

Initialement pilotée « par l'aval », par « la demande sociale », l'hydrologie urbaine est née dans les pays industrialisés, à la fin des années 60. Il s'agissait alors, face aux croissances urbaines résultant de la montée en puissance de l'industrialisation et de la révolution des modes de production agricole, de conduire une réflexion sur les techniques d'assainissement urbain développées durant la seconde moitié du XIX ${ }^{\circ}$ siècle. Les premières recherches ont donc concerné l'évaluation des flux et volumes d'eau résultant de l'urbanisation sous l'effet des précipitations, et la définition de techniques et d'ouvrages autorisant une certaine maítrise de ces flux et volumes.

A la fin des années 70 , face à la dégradation généralisée des milieux hydriques, sous l'effet des activités anthropiques, les recherches en hydrologie urbaine ont également porté sur l'étude des flux et charges de pollution issus des espaces urbanisés, et l'élaboration de méthodes et techniques de contrôle et de réduction de ces flux et charges. Plus récemment, en particulier à la suite d'inondations urbaines catastrophiques (Nîmes, 1988; Vaison-la-Romaine, 1992), les recherches se sont également orientées vers l'étude des comportements des unités hydrologiques urbaines soumises à des précipitations extrêmes.

Discipline à caractère fortement expérimental, l'hydrologie urbaine s'est appuyée, et s'appuie, sur l'observation des processus hydrologiques au sein d'unités expérimentales (bassins versants, ouvrages spécifiques). Les contraintes propres à ces observations (faibles pas de temps, accessibilité, agressivité de certains effluents, sédiments et détritus transportés par les écoulements...) ont conduit au développement de métrologies spécifiques, ou à l'adaptation de métrologies existantes aux conditions particulières de l'Hydrologie urbaine (Bertrand-Krajewski et al., 2000).

Par ailleurs, s'agissant d'étudier les relations souvent conflictuelles entre le cycle de l'eau et l'urbanisation, manifestation concentrée de l'activité anthropique, I'hydrologie urbaine a naturellement donné lieu à des ouvertures vers les sciences sociales, qu'il s'agisse, par exemple, d'analyser le rôle des comportements sociaux sur la nature des flux polluants provenant des agglomérations (eaux usées et pluviales) ou face aux risques d'inondation.

Aujourd'hui, on pourrait dire que l'hydrologie urbaine relève des sciences de l'environnement. A ce titre, ses recherches font appel à de multiples disciplines scientifiques plus ou moins $\alpha$ dures $)$. Elle constitue ainsi un intéressant laboratoire d'apprentissage de la transdisciplinarité. A contrario, elle entre difficilement dans les classifications scientifiques usuelles des organismes français de recherche. Une encyclopédie lui a été récemment consacrée, attestant de ce caractère transdisciplinaire (Chocat, 1997).

\section{3}

\section{Orientations des recherches en hydrologie urbaine}

La structuration scientifique de l'hydrologie urbaine s'est accompagnée, au début des années 80 , de la mise en réseaux, aux niveaux national et mondial, des équipes de recherche œuvrant dans la discipline. Ces réseaux diffusent des informations sur les thèmes de recherche en cours et organisent périodiquement des rencontres nationales et internationales faisant le point des avancées et des orientations scientifiques des recherches. Ainsi, en 1981, fut créé le Comité joint hydrologie urbaine (CJHU/JCUSD), commun à l'Association internationale de recherches hydrauliques (AIRH/IAHR) et à l'Association internationale de l'eau (AIE/TWA). Le Comité anime des groupes thématiques, édite une lettre de liaison et organise, tous les trois ans, une conférence internationale. La dernière s'est tenue en septembre 2002 à Portland (USA).

En France, diverses associations scientifiques ou professionnelles organisent des colloques et séminaires. Depuis 1992, a lieu, tous les trois ans, la conférence internationale NOVATECH, qui fait le point sur les avancées technologiques en assainissement pluvial (NOVATECH, 2001 ; Brelot et al., 2002). Depuis peu, les chercheurs et techniciens en hydrologie urbaine disposent également d'une revue internationale spécifique : Urban Water (coordonnateurs C. Maksimovic et D. Butler, éditeur Elsevier, GB).

Ces diverses associations, manifestations et publications permettent une évaluation des orientations des recherches. Celles-ci concernent aussi bien des aspects fondamentaux que des aspects finalisés. Les aspects fondamentaux ont trait à la connaissance et à la caractérisation des phénomènes élémentaires du cycle de l'eau en milieu urbain. Si de nombreuses recherches sur les mécanismes de transformation des précipitations en ruissellement sur les surfaces urbaines et écou- 
lement dans les systèmes d'assainissement ont permis de jeter les bases de modèles aujourd'hui largement utilisés en ingénierie (progiciels de projet, simulation ou gestion), de nombreuses recherches fondamentales sont envisageables. Elles concernent:

- l'adéquation des modélisations aux changements d'échelles spatio-temporelles :

- la modélisation des écoulements dans les secteurs urbanisés soumis à des inondations pluviales ;

- la modélisation hydrodynamique des ouvrages « spéciaux y des systèmes d'assainissement, des dispositifs d'engouffrements aux exutoires ;

- la connaissance et la mesure des précipitations en site urbain, etc.

Par ailleurs, de nombreuses recherches ầ caractère fondamental pourraient concerner les mécanismes biophysico-chimiques de transfert et de transformation des flux polluants véhiculés par temps de pluie sur les unités hydrologiques urbaines et dans leurs systèmes d'assainissement. Si de multiples recherches ont traité, ces dix dernières années, de la caractérisation et de la migration des matières en suspension et des sédiments des systèmes d'assainissement, et si d'autres études peuvent être entreprises sur ces types de polluants, d'autres recherches pourraient concerner les transformations biochimiques s'opérant dans les ouvrages et notamment ceux s'apparentant aux techniques dites ( alternatives » ou « compensatoires ») (bassins de retenue, d'infiltration, tranchées drainantes, chaussées poreuses, etc.).

Les aspects finalisés sont également nombreux et les orientations des recherches peuvent se décliner selon de multiples axes, avec, en dénominateur commun, une vision " intégrée » du cycle urbain de l'eau s'inscrivant dans le concept de « développement durable m. Ce concept est en effet essentiel dans un domaine où la durée de vie des aménagements est considérable. Sans viser à l'exhaustivité, on peut citer, parmi les axes majeurs:

- l'adaptation et la gestion des systèmes d'assainissement existants aux concepts de gestion intégrée et de développement durable. Cet axe est générateur de recherches innovantes, qu'il s'agisse de techniques de restauration ou d'adaptation des ouvrages et de leurs environnements, ou du développement de la gestion en temps réel à buts multiples ;

- la définition de méthodes et d'ouvrages s'inscrivant dans les deux concepts précédents. A ce titre, le contrôle à la source des polluants véhiculés par le ruissellement urbain constitue un thème important de recherche. De même les techniques de gestion des flux d'eau ou (et) charges polluantes, ou Best Management Practices (BPM) des Anglo-Saxons, constituent des thèmes à explorer. L'American Society of Civil Engineers en tient une évaluation périodique. A l'heure actuelle, cette association a recensé plus de 150 BPM... ;

- la prise en compte des dimensions socio-économiques et socioculturelles dans le développement d'une gestion intégrée du cycle urbain de l'eau, englobant non seulement la ville et ses ouvrages d'assainissement, mais également les milieux hydriques récepteurs superficiels et souterrains intra et extra-muros. II s'agit là d'actions « transversales $»$ de recherches finalisées en hydrologie urbaine, conséquences de l'évolution de la discipline. Ces aspects sont particulièrement importants dans le cas des pays en développement, confrontés à des croissances urbaines et à des conditions socio-économiques spécifiques. Dans ces pays, les recherches en sont encore à leurs balbutiements et il y a là matière à des collaborations solidaires dont l'ampleur pourrait être significative.

\section{4 \\ Conclusion}

Discipline scientifique et technique initialement "pilotée» par l'aval, l'hydrologie urbaine est aujourd'hui à l'origine de ses propres recherches. Elles visent à la connaissance des phénomènes mis en jeu dans le cycle de l'eau en milieu urbain et à rendre plus équilibrées les relations souvent conflictuelles entre ce cycle et l'urbanisation, et ceci au travers d'une gestion intégrée du cycle et dans un concept de développement durable. Désormais science de l'environnement à caractère fortement transdisciplinaire, elle offre de multiples perspectives de recherche s'inscrivant, notamment, dans ce dernier concept.

\section{Bibliographie}

Bertrand-Krajewski J.L., Laplace D., Joannis C., Chebbo G. - Mesures en hydrologie urbaine et assainissement, Tec et Doc Lavoisier, Paris, 2000, 794 p.

Brelot E., Chocat B., Desbordes M. (ed.) -
"Innovative technologies in urban drainage (NOVATECH 2001) x. Water Science and Technology, 45 (7), 2002.

Chocat B. (ss la dir.) - Encyclopédie de Ihydrologie urbaine et de l'assainisse- ment. Tec et Doc Lavoisier. Paris, 1997, $1124 \mathrm{p}$.

NOVATECH - Les nouvelles technologies en assainissement pluvial. GRAIE/INSA de Lyon, 2001, 1128 p. 